\title{
Recovering real-world images from single-scale boundaries with a novel filling-in architecture
}

\author{
Matthias S. Keil ${ }^{\mathrm{a}, *}$, Gabriel Cristóbal ${ }^{\mathrm{b}}$, Thorsten Hansen ${ }^{\mathrm{c}}$, Heiko Neumann ${ }^{\mathrm{d}}$ \\ ${ }^{\mathrm{a}}$ Centre de Visió per Computador, Edifici O, Campus UAB, E-08193 Bellaterra (Cerdanyola), Barcelona, Spain \\ ${ }^{\mathrm{b}}$ Instituto de Óptica (CSIC), Image and Vision Department, E-28006 Madrid, Spain \\ ${ }^{\mathrm{c}}$ Universität Giessen, Department of Psychology, D-35394 Giessen, Germany \\ ${ }^{\mathrm{d}}$ Universität Ulm, Abteilung Neuroinformatik, D-89069 Ulm, Germany
}

Received 24 November 2002; accepted 16 April 2005

\begin{abstract}
Filling-in models were successful in predicting psychophysical data for brightness perception. Nevertheless, their suitability for real-world image processing has never been examined. A unified architecture for both predicting psychophysical data and real-world image processing would constitute a powerful theory for early visual information processing. As a first contribution of the present paper, we identified three principal problems with current filling-in architectures, which hamper the goal of having such a unified architecture. To overcome these problems we propose an advance to filling-in theory, called BEATS filling-in, which is based on a novel nonlinear diffusion operator. BEATS filling-in furthermore introduces novel boundary structures. We compare, by means of simulation studies with real-world images, the performance of BEATS filling-in with the recently proposed confidence-based filling-in. As a second contribution we propose a novel mechanism for encoding luminance information in contrast responses ('multiplex contrasts'), which is based on recent neurophysiological findings. Again, by simulations, we show that 'multiplex contrasts' at a single, high-resolution filter scale are sufficient for recovering absolute luminance levels. Hence, 'multiplex contrasts' represent a novel theory addressing how the brain encodes and decodes luminance information.
\end{abstract}

(C) 2005 Elsevier Ltd. All rights reserved.

Keywords: Filling-in models; Real-world image; BEATS filling-in

\section{Introduction}

In the natural environment, surface boundaries are often defined by luminance contrasts indicating changes in material. Experimental data indicate that such boundaries are represented in the visual system: contrast sensitive neurons are already present after the photoreceptor level in the retina (Kuffler, 1953; Werblin \& Dowling, 1969), and in the primary visual cortex (Hubel \& Wiesel, 1962, 1968). However, our perception is richer than mere boundary maps, and there is now evidence that cortical neurons exist whose responses correlate with surface brightness (e.g. Komatsu, Murakami, \& Kinoshita, 1996; Kinoshita \& Komatsu, 2001; MacEvoy, Kim, \& Paradiso, 1998; Rossi,

\footnotetext{
* Corresponding author

E-mail address: mats@cvc.uab.es (M.S. Keil).
}

0893-6080/\$ - see front matter (C) 2005 Elsevier Ltd. All rights reserved. doi:10.1016/j.neunet.2005.04.003
Rittenhouse, \& Paradiso, 1996; Sasaki \& Watanabe, 2004). Given that the retina transmits contrast information to the cortex, and the evidence for boundary sensitive neurons in the cortex, one may ask how the visual system assigns perceptual attributes (such as brightness) to surfaces. As a possible solution it was suggested that contrasts subserve boundary detection, and that contrast activity fills into areas enclosed by boundaries, thereby generating surface representations (Walls, 1954). Thus, surface representations are generated at their boundaries by filling-in local contrast information. A first framework for filling-in was proposed by Gerrits and Vendrik (1970), for explaining data on stabilized retinal images (Gerrits, de Haan, \& Vendrik, 1966). Filling-in within this framework occurs in separate channels for 'brightness' and 'darkness' activity, corresponding to retinal $\mathrm{ON}$ and OFF contrast channels, respectively (Fig. 1). Brightness activity determines the surface attribute 'brighter than mid-gray', and darkness activity determines the attribute 'darker than mid-gray'. 


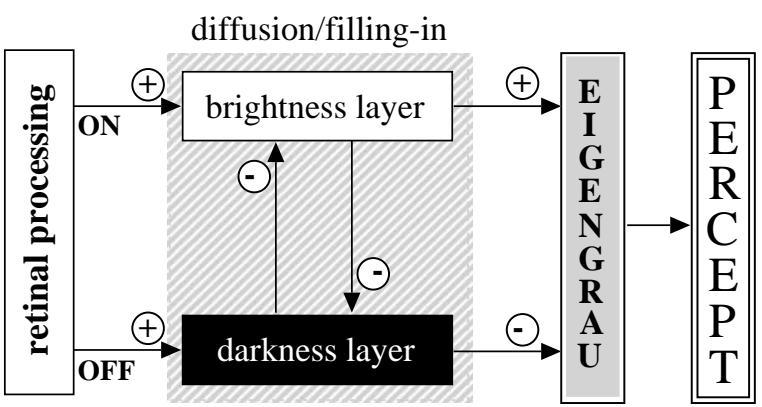

Fig. 1. Filling-in framework proposed by Gerrits and Vendrik (1970). Retinal ON (OFF)-responses provide the initial state in a brightness (darkness) diffusion layer. Activity propagation is controlled by mutual inhibition of spatially corresponding cells in both layer. Perceived luminance is computed by brightness (darkness) activity acting excitatory (inhibitory) on an Eigengrau level (Knau \& Spillman, 1997; Gerrits \& Vendrik, 1970).

The mid-gray level is called Eigengrau (Gerrits \& Vendrik, 1970; Knau \& Spillman, 1997), and refers to the percept which is reported by subjects after the disappearance of a stabilized retinal image. Here, we will use the term perceived luminance or perceptual activity to describe the final appearance of a surface. Perceived luminance is assumed to be a function of brightness activity and darkness activity. ${ }^{1}$

The filling-in framework proposed by Gerrits and Vendrik was formalized by Cohen and Grossberg (1984) and Grossberg and Mingolla (1985) (herein referred to as standard filling-in). Standard filling-in clarified the role of boundaries and features by proposing two interacting subsystems (see Fig. 2), a boundary contour system (BCS), and a feature contour system (FCS).

With standard filling-in, a given intensity image is initially processed by center-surround mechanisms, modeling retinal contrast enhancement (Marr \& Hildreth, 1980; Marr, 1982). Center-surround processing results in two information channels, one for conveying ON-contrasts (signalling luminance increments), and one for OFFcontrasts (signalling luminance decrements). Both, FCS and BCS, make use of these contrast channels. BCS processing implements an edge detector by looking for spatially coinciding ON and OFF contrast patterns. Edges define areas to be filled-in by the FCS. Filling-in is implemented by heat diffusion, ${ }^{2}$ whereby BCS edges act

\footnotetext{
${ }^{1}$ Usually, in the literature it is referred to as (perceived) brightness to what we mean with perceived luminance, and perceptual activity, respectively. We introduced the latter terms to avoid confusion, since we further distinguish brightness related activity, and a darkness related activity.

${ }^{2}$ Filling-in may appear similar to anisotropic diffusion models (Perona \& Malik, 1990) at first sight. However, diffusion in filling-in models occurs isotropically, using a scale-constant boundary structure (see also Neumann et al., 2001). Since filling-in models aim to explain psychophysical and neurophysiological data, and no specific adaptive anisotropies have been reported so far, models remain at using isotropic completion mechanisms for surface attributes.
}

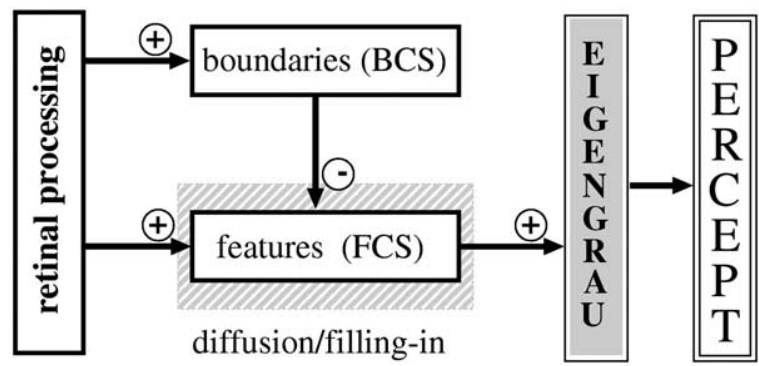

Fig. 2. Filling-in framework proposed by Cohen and Grossberg (1984). A boundary contour system (BCS) controls activity propagation in the feature contour system (FCS, the diffusion layer). Notice that boundaries have to be available before filling-in begins.

as barriers for the diffusion process (Grossberg \& Todorović, 1988).

Various experiments corroborate the filling-in hypothesis, both behaviorally (Davey, Maddess, \& Srinivasan, 1998a; Davey, Srinivasan, \& Maddess, 1998b; Paradiso \& Nakayama, 1991; Paradiso \& Hahn, 1996; Watanbade \& Sato, 1989;) and neurophysiologically (Kinoshita \& Komatsu, 2001; MacEvoy et al., 1998; Rossi et al., 1996; Rossi \& Paradiso, 1999, but see Pessoa \& Neumann, 1998, and Pessoa, Thompson, \& Noë, 1998). Corresponding models evolved in parallel, where models addressed, for example, dynamical aspects (Arrington, 1994; Grossberg, Francis, \& Mingolla, 1994; Keil \& Neumann, 2001), the processing of luminance gradients (Grossberg \& Mingolla, 1987; Pessoa, Mingolla, \& Neumann, 1995), perceptual grouping (Gove, Grossberg, \& Mingolla., 1995; Ross, Grossberg, \& Mingolla, 2000), and the three-dimensional perception of surfaces (Grossberg, 1997; Grossberg \& Pessoa, 1998; Grossberg \& Howe, 2003; Kelly \& Grossberg, 2000). Filling-in models were also applied to improve the perceived quality of synthetic aperture radar (SAR) images (Grossberg, Mingolla, \& Williamson, 1995; Mingolla, Ross, \& Grossberg, 1999). But for all that success so far, results from filling-in models with real-world images remain scarce (with the exception of Hong and Grossberg (2004) and Sepp \& Neumann (1999)).

The first goal of our paper was to investigate why there exists such discrepancy between explaining perceptual data and the apparent limitations of filling-in models when applied to real-world data. We identified three problems: (i) The introduction of gradients into the perceptual activity of surface representations, which were actually absent from the visual input (trapping problem). (ii) Filled-in representations often suffer from a loss of contrast compared to the input (fogging problem). (iii) Originally sharp-edged surfaces in the visual input are mapped onto surface representations with blurred edges (blurring problem; see, for example, simulation results of Gove et al., 1995; Grossberg et al., 1995; Mingolla et al., 1999; Sepp \& Neumann, 1999).

We further asked how to reduce those artifacts, and present an advance to filling-in theory, which we called 


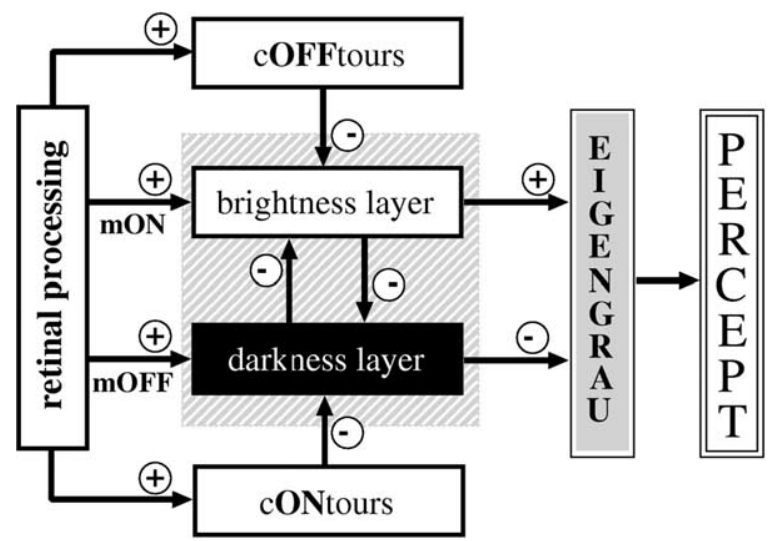

Fig. 3. Filling-in framework proposed in this paper. Instead of having a single BCS for both diffusion layers, each layer possesses its own set of boundaries (i.e. contours and cofftours). The diffusion process is nonlinear, because of using a nonlinear diffusion operator, and mutual inhibition of brightness and darkness layer in an interaction zone. This interaction was introduced since boundary gaps have more deleterious effects due to the nonlinear diffusion operator.

BEATS filling-in ('Bigger EATs Smaller', Fig. 3). Unlike its predecessors, BEATS filling-in is based on a nonlinear diffusion operator. The dynamics of the corresponding diffusion process is such that smaller activity values are overwritten, or 'eaten', by bigger ones. BEATS filling-in furthermore introduces a novel boundary structure in order to enhance the spatial accuracy for controlling the diffusion process. Whereas usually a single set of boundaries slows down diffusion in both the brightness and the darkness diffusion layer, BEATS filling-in uses two sets of boundaries, which are called contours (darkness layer) and cofftours (brightness layer). In conjunction with nonlinear diffusion, the novel boundary structure counteracts the blurring and trapping problem.

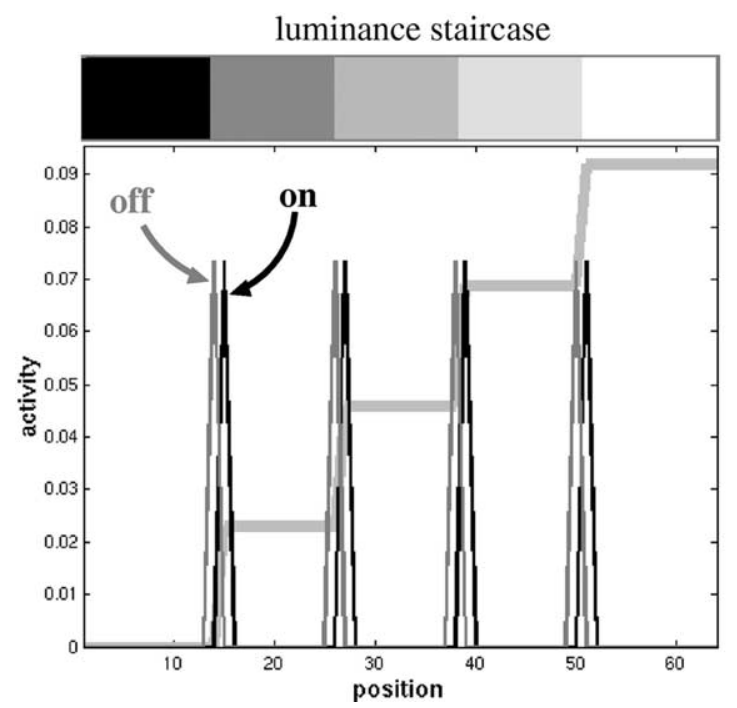

As a second contribution of this paper we propose a new mechanism of how luminance information is made available in the visual cortex. Retinal ganglion cells are sensitive to luminance contrast within their receptive field (Kaplan \& Shapley, 1982; Kaplan, Lee, \& Shapley, 1990; Kuffler, 1953). However, by considering only contrast information, we cannot distinguish the stimuli shown in Fig. 4. As a solution, most models addressing brightness perception and image coding, respectively, propose a low-pass or luminance channel, in addition to (often multi-scale) contrast channels (e.g. du Buf \& Fischer, 1995; Hong \& Grossberg, 2004; Pessoa et al., 1995, but see Arrington, 1996): luminance information is superimposed with filled-in or multi-scale-contrast information to recover absolute luminance levels.

Recently, a luminance-sensitive ganglion cells (GC) has been reported which projects to the suprachiasmatic nucleus (SCN) (Berson, Dunn \& Takao, 2002). This type of ganglion cell (SCN-GC) is thought to be involved in entraining circadian rhythms. However, it is an unlike candidate for a luminance-driven channel, because it does not use rod nor cone input (but is rather excited by its lightsensitive dendrites). Furthermore, the response kinetics of SCN-GCs is far too sluggish for normal vision.

Another candidate, the biplexiform cell (Mariani, 1982; Zrenner, Nelson, \& Mariani, 1983), uses direct input from rod photoreceptors. However, the function of biplexiform cells is poorly understood, and it is hypothesized that they may be involved in controlling pupil size, or in entraining circadian rhythms.

Since we could not found evidence in the literature for other mechanisms which support the notion of a luminancedriven channel, we propose instead that luminance modulates local contrast responses in a way that contrast

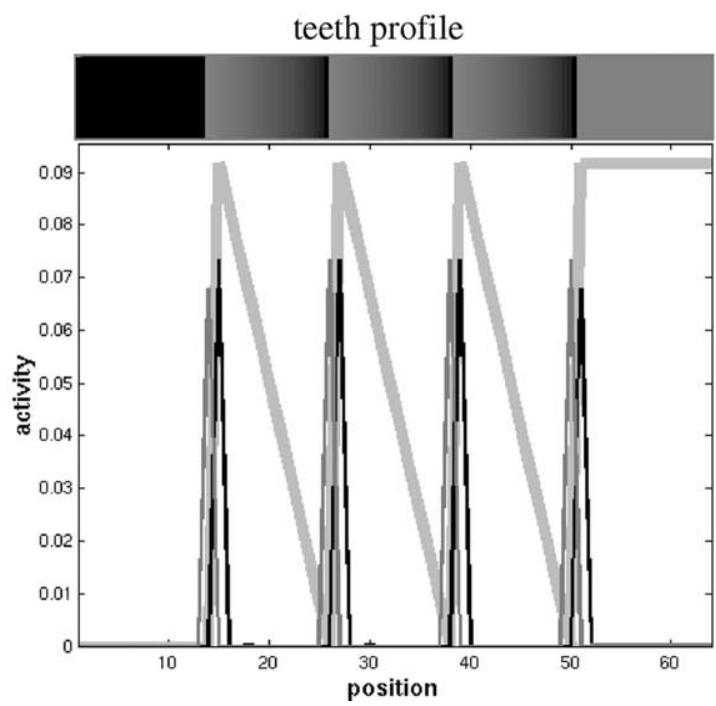

Fig. 4. Contrasts alone are ambiguous. By only considering contrast information, one cannot distinguish a luminance staircase (left) from a teeth-shaped luminance profile (right). Thin curves represent retinal ON and OFF-responses, bold gray curves represent luminance (2D luminance distributions are shown above each plot). 
and luminance are simultaneously encoded (multiplex code). Then, filling-in of multiplex contrasts directly recovers an anchoring level of perceived luminance based on absolute luminance levels. Notice that, in order to recover an anchoring level with a luminance-driven channel, one has to add its output to the output of filling-in layers (i.e. after filling-in has been completed), what implies that filling-in alone is insufficient for the generation of brightness maps.

The notion of a multiplex code involves a consistent interpretation of anatomical evidence provided by $\mathrm{Li}$, Pei, Zhou, and von Mitzlaff (1991) and Li, Zhou, Pei, Qiu, Tang, and $\mathrm{Xu}$ (1992), and is also in line with recent evidence demonstrating a fast and low-level mechanism for encoding human brightness information (McCourt \& Foxe, 2004).

In this paper we present simulations with the novel BEATS filling-in, and the recently propose confidencebased filling-in (Neumann, Pessoa, \& Hansen, 2001). We show that brightness of real-world images can be recovered by using only single-scale high-pass information. The possible impact of our results on human brightness coding and encoding of images is discussed.

\section{Retinal model}

Whereas previous filling-in models use the same contrast information for boundary detection and filling-in, we use to different types of contrasts, as illustrated in Fig. 3. Retinal contrasts for boundary detection correspond to high-pass information and were computed with a dynamic normalization network (details are given in Keil, 2003). Unlike spatial differentiation with a Laplacian, our approach leads to 'smoother' boundary structures at feature junctions. Contrasts for filling-in were computed with a Laplacian-like receptive field structure (Marr \& Hildreth, 1980).

The center/surround receptive field of our model ganglion cells is specified as follows. Center width was one pixel, that is $C \equiv \mathcal{L}$, where $\mathcal{L} \in[0,1]$ is the input image. Surround activity was computed by convolving $\mathcal{L}$ with a $3 \times 3$ kernel with zero center weight, $\exp (-1) / \eta$ for the four nearest neighbors, and $\exp (-2) / \eta$ for the four nextnearest neighbors ( $\eta$ was chosen such that the kernel integrated to one). Retinal responses were evaluated at steady-state of

$$
\begin{aligned}
\frac{\mathrm{d} V_{i j}(t)}{\mathrm{d} t}= & g_{\text {leak }}\left(V_{\text {rest }}-V_{i j}\right)+E_{\text {cent }}^{\prime} e_{i j}+E_{\text {surr }}^{\prime} \mathcal{S}_{i j} \\
& +\mathscr{I}_{i j, s i}\left(E_{s i}-V_{i j}\right)
\end{aligned}
$$

with parameter values specified in Table 1 . The decay of activity is defined by $g_{\text {leak }}$, and $V_{\text {rest }}$ is a resting potential. The term $\mathscr{I}_{i j, s i} \equiv\left[E_{\text {cent }}^{\prime} \mathcal{C}_{i j}+E_{\text {surr }}^{\prime} \mathcal{S}_{i j}\right]^{+}$denotes self-inhibition with reversal potential $E_{s i}$, where $[\cdot]^{+} \equiv \max (\cdot, 0)$.

Self-inhibition implements the compressive and nonlinear response curve observed in biological X-type cells (Kaplan et al., 1990). ON-responses $x^{\otimes} \equiv[V]^{+}$are obtained
Table 1

Parameter values for the retinal stage. Notice that Eq. (1) actually represents two equations (for $\mathrm{ON}$ - and OFF-contrasts), defined by the

\begin{tabular}{|c|c|c|c|}
\hline Parameter & Value & Equation & Description \\
\hline$g_{\text {leak }}$ & 1 & 1 & Leakage conductance \\
\hline$V_{\text {rest }}$ & 0 & 1 & Resting potential \\
\hline$E_{\mathrm{si}}$ & 0 & 1 & $\begin{array}{l}\text { Self-inhibition reversal } \\
\text { potential }\end{array}$ \\
\hline$\left(E_{\text {cent }}^{\prime}, E_{\text {surr }}^{\prime}\right)$ & $(1,-1)$ & 1 & $\begin{array}{l}\text { ON-cell (center, surround) } \\
\text { amplitude }\end{array}$ \\
\hline$\left(E_{\text {cent }}^{\prime}, E_{\text {surr }}^{\prime}\right)$ & $(-1,1)$ & 1 & $\begin{array}{l}\text { OFF-cell (center, surround) } \\
\text { amplitude }\end{array}$ \\
\hline $\mathrm{D}$ & 0.35 & 2 & Saturation constant \\
\hline$\sigma_{\mathrm{OS}}$ & 4 & 2 & $\begin{array}{l}\text { Outer surround spatial } \\
\text { constant }\end{array}$ \\
\hline
\end{tabular}
respective values of $E_{\text {cent }}^{\prime}$ and $E_{\text {surr }}^{\prime}$

by setting $E_{\text {cent }}^{\prime}=1$ and $E_{\text {surr }}^{\prime}=-1$, and OFF-responses $x^{\otimes} \equiv[V]^{+}$by $E_{\text {cent }}^{\prime}=-1$ and $E_{\text {surr }}^{\prime}=1$. With Eq. (1), ONand OFF-responses at luminance edges have equal amplitudes (c.f. Fig. 4). ${ }^{3}$

Multiplexed retinal responses $\tilde{m}^{\oplus}$ and $\tilde{m}^{\ominus}$ were generated by modulating $x^{\oplus}$ and $x^{\ominus}$, respectively, with average local luminance. Averaging took place within a region - called outer surround $\mathcal{O S}$ - which is a structure situated beyond the classical receptive field of retinal ganglion cells ( $\mathrm{Li}$ et al., 1991; Li et al., 1992; see Fig. 10).

Let $\operatorname{Norm}[\cdot]$ implement the normalization operator, that is if $a \leq f_{i j} \leq b \forall i, j$, and $a \neq b$, then $0 \leq \operatorname{Norm}\left[f_{i j}\right] \leq 1$. (The normalization operator represents a simple model for network adaptation to the full dynamic range of the input) . The activity of the outer surround is computed by convolution (symbol ' $\otimes$ ') with a Gaussian kernel: $\mathcal{O} \mathcal{S} \equiv \operatorname{Norm}[\mathcal{L}] \otimes \mathcal{L}_{\sigma_{O S O}}$. We chose $\sigma_{\mathrm{OS}}=4$, in line with the data from $\mathrm{Li}$ et al. $(1991,1992)$, who found that the outer surround is large compared to the 'classical' surround of ganglion cells. Nevertheless, we did not observe significant differences in our results for choosing larger or smaller values for $\sigma_{\mathrm{os}}$. Outer surround activity acts to multiplicatively gate the 'classical' center/surround responses of retinal ganglion cells according to

$\tilde{m}_{i j}^{\otimes}=x_{i j}^{\otimes} \frac{\mathcal{O} \mathcal{S}_{i j}}{D+\mathcal{O} \mathcal{S}_{i j}} \quad \tilde{m}_{i j}^{\ominus}=x_{i j}^{\ominus} \frac{1-\mathcal{O} \mathcal{S}_{i j}}{D+1-\mathcal{O} \mathcal{S}_{i j}}$

where $D$ is a saturation constant (bigger values result in a more linear encoding of luminance). For fixed contrasts, $\tilde{m}^{\oplus}$ increases as a function of intensity, and consequently $\tilde{m}^{\oplus}$ encodes local brightness. Likewise, $\tilde{m}^{\ominus}$ decreases as a function of intensity, making $\tilde{m}^{\ominus}$ encode local darkness.

\footnotetext{
${ }^{3}$ In biological ganglion cells, ON- and OFF channels are not symmetric in many properties. For instance, ON cells fire spontaneously at a higher rate than OFF cells under photopic stimulation (Chichilnisky \& Kalmar, 2002; Cleland, Levick \& Sanderson, 1973; Kaplan, Purpura, \& Shapley, 1987; Passaglia, Enroth-Cugell, \& Troy, 2001; Troy \& Robson, 1992; Zaghloul, Boahen, \& Demb, 2003).
} 


\section{A generic diffusion operator}

In this section we define a general diffusion operator for describing previously existing filling-in mechanisms as special cases. Let $f^{\circ}$ be a function denoting brightness activity, and $f^{\bullet}$ a function that encodes darkness activity. We assume the existence of two separate diffusion layers, one for brightness activity and one for darkness activity. Then, we can define diffusion operators $\mathcal{K}^{\circ}$ and $\mathscr{K}^{\bullet}$ for the brightness layer and the darkness layer, respectively, as

$\mathcal{K}_{\varepsilon, \lambda}^{\circ} f_{i j}^{\circ}=\sum_{(p, q) \in N_{i j}} P_{i j p q}^{\circ} \mathcal{T}_{\lambda}\left[f_{p q}^{\circ}-f_{i j}^{\circ}\right]$

$\mathcal{K}_{\varepsilon, \lambda}^{\bullet} f_{i j}^{\bullet}=\sum_{(p, q) \in N_{i j}} P_{i j p q}^{\bullet} \mathcal{T}_{\lambda}\left[f_{p q}^{\bullet}-f_{i j}^{\bullet}\right]$

where $\boldsymbol{N}_{i j}$ denotes a von Neumann neighborhood. ${ }^{4}$ Since boundaries block activity propagation, we define spatially varying diffusion coefficients, or permeabilities, for the brightness layer $P^{\circ} \equiv P^{\circ}\left(\Gamma^{\circ}\right)$, and the darkness layer $P^{\bullet} \equiv P^{\bullet}\left(\Gamma^{\bullet}\right)$, respectively, as

$P_{i j p q}^{\circ}\left(\Gamma^{\circ}\right)=\left(1+\varepsilon \Gamma_{i j p q}^{\circ}\right)^{-1}$

$P_{i j p q}^{\bullet}\left(\Gamma^{\bullet}\right)=\left(1+\varepsilon \Gamma_{i j p q}^{\bullet}\right)^{-1}$

Both $\Gamma^{\circ}$ and $\Gamma^{\bullet}$ are functions of boundary signals (or BCS-activity), which act as diffusion barriers. The effectiveness of blocking diffusion is characterized by $\varepsilon$. The operator $\mathcal{T}_{\lambda}[\cdot]$ is defined by

$\mathcal{T}_{\lambda}[x]=\frac{\eta x}{1+\mathrm{e}^{-\lambda x}}$

where $\eta=1+\mathrm{e}^{-|\lambda|}$ is a normalization constant. The parameter $\lambda$ defines the functional behavior of $\mathcal{T}_{\lambda}[\bullet]$, that is

1. Inverse half-wave rectification:

$\lim _{\lambda \rightarrow-\infty} T_{\lambda}[x]=\min (0, x)$

2. Identity:

$\lambda=0 \rightarrow \mathcal{T}_{0}[x]=x$

3. Half-wave rectification:

$\lim _{\lambda \rightarrow+\infty} T_{\lambda}[x]=\max (x, 0)$

Details of the derivation of the operator and its limit properties can be found in Keil, 2003.

\section{BEATS ('bigger EATs smaller') filling-in}

Owing to a nonlinear diffusion operator (Eq. (8)), the dynamics of BEATS filling-in looks as if cells with lower

\footnotetext{
${ }^{4}$ A von Neumann neighborhood $N_{i j}$ of a grid point $(i, j)$ denotes its four nearest neighbors $N_{i j}=\{(i+1, j),(i-1, j),(i, j+1),(i, j+1)\}$.
}

Table 2

Parameter values for BEATS filling-in. Eq. (13) was integrated with the forth-order Runge Kutta method (with parameter values given in the last two rows of the table)

\begin{tabular}{|c|c|c|c|}
\hline Parameter & Value & $\begin{array}{l}\text { Equati- } \\
\text { on }\end{array}$ & Description \\
\hline$\varepsilon$ & 25.0 & 3 & $\begin{array}{l}\text { contour/cofftour diffusion slow } \\
\text { down gain }\end{array}$ \\
\hline$\lambda$ & $\rightarrow \infty$ & 3 & Max-diffusion \\
\hline$\Theta_{w}$ & 0.0125 & 9 & contour/cofftour threshold \\
\hline$\beta_{w}$ & 1.0 & 9 & contour/cofftour saturation constant \\
\hline$\Theta_{z}$ & 0.070 & 10 & Interaction zone threshold value \\
\hline$\beta_{z}$ & 0.005 & 11 & Interaction zone saturation constant \\
\hline$\sigma_{z}$ & 2.0 & 11 & Interaction zone blurring constant \\
\hline$\gamma_{w}$ & 0.75 & 13 & contour/cofftour inhibitory gain \\
\hline$E_{\text {in }}$ & -0.025 & 13 & $\begin{array}{l}\text { contour/cofftour inhibitory reversal } \\
\text { activity }\end{array}$ \\
\hline$\Delta_{t}$ & 1.0 & & Forth-order Runge Kutta time step \\
\hline$t_{\max }$ & $\leq 600$ & & Number of integration time steps \\
\hline
\end{tabular}

Since only the parameters' order of magnitude matters, all simulation results are robust with respect to specific parameter values.

activities are 'eaten' by neighbors with higher activities. The nonlinear diffusion operator is thought to model rectifying (i.e. voltage dependent) gap junctions (e.g. Edwards, Heitler, Leise, \& Friscke, 1991; Edwards, Yeh, \& Krasne, 1998). BEATS filling-in is now introduced formally, with parameter values given in Table 2. A sketch of the architecture is shown in Fig. 3.

\subsection{Contours and cofftours}

To keep the model simple, we did not explicitly model the computation of boundary maps with oriented contrast operators, what would, in cortical terms, correspond to compute simple and complex cell responses, respectively. Instead, we estimated the latter responses by retinal $\mathrm{ON}$ - and OFF-responses (denoted by $\hat{y}^{\oplus}$ and $\tilde{y}^{\ominus}$, respectively). Those estimates are contours (activity $w^{\circ}$ ) representing diffusion barriers in the darkness diffusion layer, and cofftours (activity $w^{\bullet}$ ) acting as diffusion barriers in the brightness diffusion layer (see Fig. 5):

$w_{i j}^{\circ}=\frac{\operatorname{thresh}_{\Theta_{w}}\left(\tilde{y}_{i j}^{\oplus}\right)}{\beta_{w}+\operatorname{thresh}_{\Theta_{w}}\left(\hat{y}_{i j}^{\oplus}\right)}$

$w_{i j}^{\bullet}=\frac{\operatorname{thresh}_{\Theta_{w}}\left(\tilde{y}_{i j}^{\ominus}\right)}{\beta_{w}+\operatorname{thresh}_{\Theta_{w}}\left(\tilde{y}_{i j}^{\ominus}\right)}$

where thresh $_{\Theta_{w}}(x)=\left[\operatorname{Norm}[x]-\Theta_{w}\right]^{+}$implements adaptive thresholding with threshold $\Theta_{w} \in[0,1]$. This threshold is associated with gradient suppression and smoothness of filledin surface representations. $\beta_{w}$ is a saturation constant for amplifying supra-threshold boundary activity. The computation of boundary contrasts $\tilde{y}^{\oplus}$ and $\tilde{y}^{\ominus}$ has been outlined in Section 2. The motivation for using two sets of boundary is described in Section 6.1. Notice that we use a steady boundary structure while diffusion proceeds. This is different to 

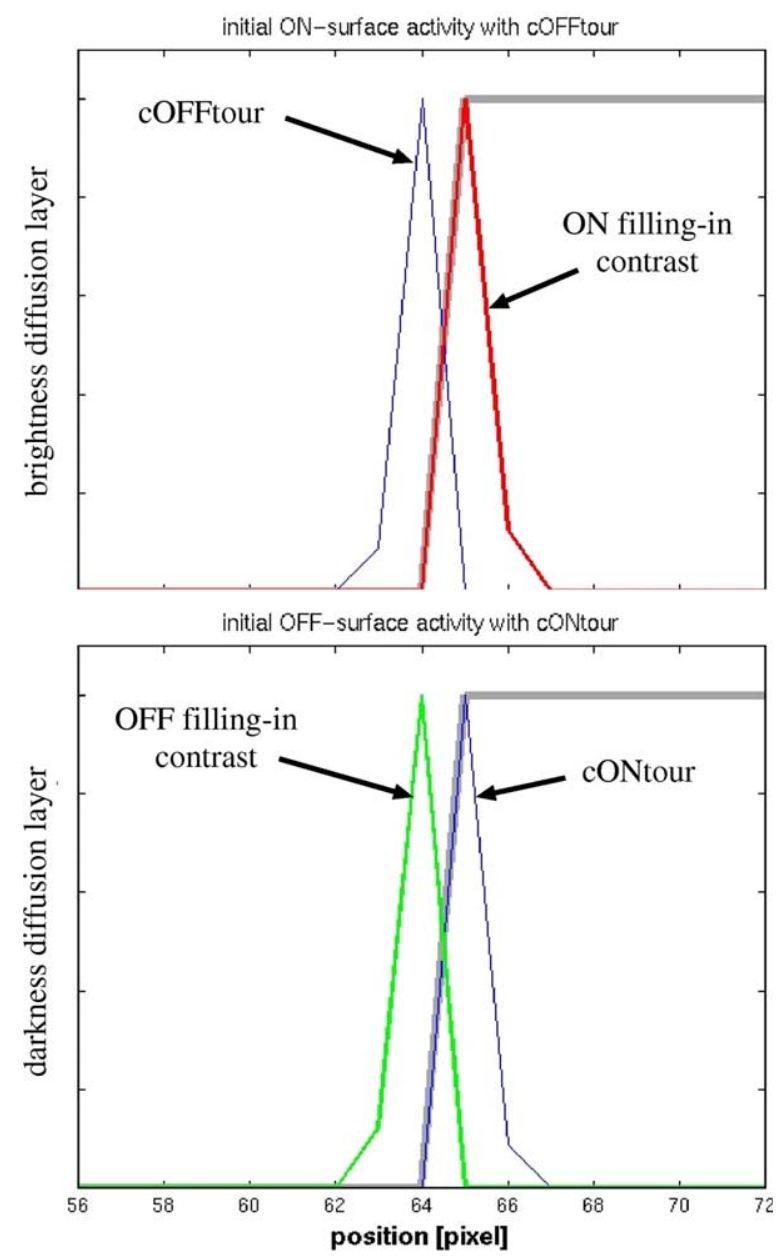

Fig. 5. Boundaries and filling-in contrasts do not overlap with BEATS filling-in. BEATS filling-in uses two sets of boundaries (contours and cofftours) such that filling-in contrasts cannot overlap with boundaries. In this way activity trapping is avoided. Bold gray curves correspond to luminance. anisotropic diffusion approaches, where boundaries traverse the scale space with time (Perona \& Malik, 1990).

\subsection{Brightness/darkness interaction zone}

A boundary with 'holes' (i.e. a not entirely 'watertight' boundary) allows for activity exchange between adjacent surface representations. As a consequence, perceived luminance contrasts between surfaces decrease, because they eventually adopt the same value of perceptual activity ('fogging'). In order to counteract fogging, we define an interaction zone around contours and cofftours. Within this zone, brightness activity and darkness activity undergo mutual (divisive) inhibition. This causes a slow-down of diffusion rate at boundary leaks (since then brightness and darkness will spatially coincide). Thus, fogging is decelerated, and surface edges will appear blurry at boundary gaps. Let

$z_{i j}=\operatorname{thresh}_{\Theta_{\mathrm{z}}}\left(w_{i j}^{\circ}+w_{i j}^{\bullet}\right)$

with a threshold value $\Theta_{z}$. Interaction zone activity $\mathscr{Z}$ is defined as

$\mathscr{L}=\frac{z}{\beta_{z}+z} \otimes \mathscr{L}_{\mathrm{z}}$

where $\beta_{z}$ is a saturation constant for amplifying small values of boundary activity, and $\sigma_{z}$ the standard deviation of the Gaussian kernel $\mathscr{L}_{\sigma_{\mathrm{z}}}$ (' $\otimes$ ' denotes spatial convolution). Why not extending the interaction zone across the whole surface? Because doing so would increase significantly the required time to arrive at homogeneously filled-in surface representations in cases where brightness and darkness activity spatially overlap during filling-in. The luminance staircase shown in Fig. 6 constitutes a corresponding situation.

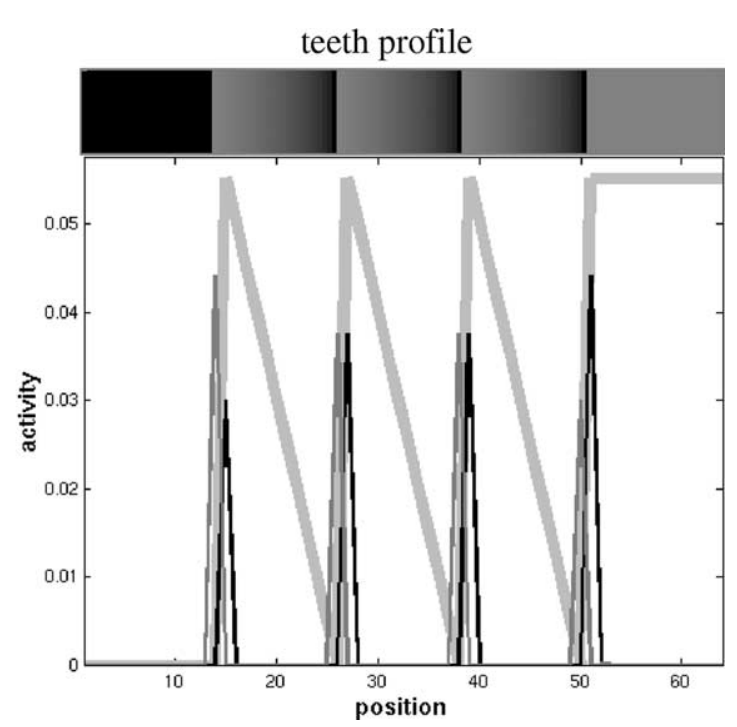

Fig. 6. Multiplexing luminance with contrast. By modulation of contrast responses according to local intensity within an outer surround (Fig. 10), it is now possible to distinguish between the luminance staircase and the teeth-shaped luminance profile by means of contrast responses. 


\subsection{Nonlinear diffusion operator}

BEATS filling-in employs max-diffusion, which corresponds to $\lambda \rightarrow \infty$ in Eq. (3). The permeabilities $P^{\circ} \equiv P^{\circ}\left(\Gamma^{\circ}\right)$ and $P^{\bullet} \equiv P^{\bullet}\left(\Gamma^{\bullet}\right)$ are instantiated by defining the functions $\Gamma^{\circ}$ and $\Gamma^{\bullet}$ of Eq. (4) as

$\Gamma_{i j p q}^{\circ}=\mathscr{L}_{i j}\left[f_{i j}^{\bullet}\right]^{+}+\mathscr{L}_{p q}\left[f_{p q}^{\bullet}\right]^{+}$

$\Gamma_{i j p q}^{\bullet}=\mathscr{L}_{i j}\left[f_{i j}^{\circ}\right]^{+}+\mathscr{L}_{p q}\left[f_{p q}^{\circ}\right]^{+}$

where $[\cdot]^{+}$denotes half-wave rectification.

\subsection{Brightness/darkness diffusion layer}

Diffusion layers are defined as

$$
\begin{aligned}
& \frac{\mathrm{d} b_{i j}^{\circ}(t)}{\mathrm{d} t}=\gamma_{w} w_{i j}^{\bullet}\left(E_{i n}-b_{i j}^{\circ}\right)+\mathcal{K}_{\varepsilon, \infty}^{\circ} b_{i j}^{\circ}+\delta\left(t-t_{0}\right) \tilde{m}_{i j}^{\oplus} \\
& \frac{\mathrm{d} b_{i j}^{\bullet}(t)}{\mathrm{d} t}=\gamma_{w} w_{i j}^{\circ}\left(E_{i n}-b_{i j}^{\bullet}\right)+\mathcal{K}_{\varepsilon, \infty}^{\bullet} b_{i j}^{\bullet}+\delta\left(t-t_{0}\right) \tilde{m}_{i j}^{\ominus}
\end{aligned}
$$

with $b^{\circ}\left(b^{\bullet}\right)$ denoting brightness (darkness) activity. The initial state $\left(t=t_{0}\right)$ of the diffusion layers is defined by multiplexed activities $\tilde{m}^{\oplus}$ and $\tilde{m}^{\ominus}$, respectively. Layer cells are assumed to have zero activity for $t \leq t_{0}$. In order to prevent activity propagation across contours and cofftours, we took advantage of the fact that max-diffusion cannot propagate negative activity values. Consequently, we let contours and cofftours hyperpolarize layer cells with weight $\gamma_{w}$. The lower hyperpolarization limit is defined by $E_{i n}$. Output activities are $S^{\circ} \equiv\left[b^{\circ}\right]^{+}$, and $S^{\bullet} \equiv\left[b^{\bullet}\right]^{+}$.

\subsection{Perceptual activity of surface representations}

The perceived activity of filled-in surface representations correspond to the non-rectified steady-state activity

$p_{i j}=\frac{g_{\text {leak }} V_{\text {rest }}+E_{e x} S_{i j}^{\circ}+E_{i n} S_{i j}^{\circ}}{g_{\text {leak }}+S_{i j}^{\circ}+S_{i j}^{\bullet}}$

with parameters given in Table 3 The Eigengrau level (Gerrits \& Vendrik, 1970; Knau \& Spillman, 1997) is set to $V_{\text {rest }}=0$. The last equation applies to both BEATS filling-in and confidence-based filling-in.

Table 3

Parameter values for the perceptual stage

\begin{tabular}{lcll}
\hline Parameter & Value & Equation & Description \\
\hline$g_{\text {leak }}$ & 1 & 14 & Leakage conductance \\
$V_{\text {rest }}$ & 0 & 14 & $\begin{array}{l}\text { Resting potential } \\
\text { (Eigengrau value) }\end{array}$ \\
$E_{\text {ex }}$ & 1 & 14 & Maximum brightness value \\
$E_{\text {in }}$ & -1 & 14 & Maximum darkness value \\
\hline
\end{tabular}

Notice that Eq. (14) is just a means for visualizing the output of the model, and does not represent a full anchoring mechanism (see e.g. Gilchrist et al., 1999).

\section{Confidence-based filling-in}

We compare the newly proposed filling-in mechanism with previously developed approaches. Therefore, we present a brief summary introduction for the recently regularized mechanism of confidence-based filling-in. We utilize the notations developed in the previous sections, thus showing the generalization properties of the new scheme.

Standard filling-in generates non-uniformly, or 'bowed', surface representations: activity is maximal at the sites of filling-in contrasts (i.e. at boundaries), and decreasing while moving further into the middle of a surface. This 'bowing effect' is more pronounced for larger surfaces. To examine this artifact Neumann et al. (2001) investigated filling-in and identified the nature of the inverse problem. They showed that standard filling-in tries to 'fit' its activity values to 'zero data' at positions where there are actually no data measurements available (this is equivalent to minimizing everywhere the data term of a regularization functional). This minimization is due to a spatially constant activity decay on the one hand, and due to 'clamped' retinal fillingin contrasts on the other. Neumann et al. accordingly modified the equations for the diffusion layers such that both the passive decay $g_{\text {leak }}$, and the 'clamped' contrasts (here $\tilde{m}^{\oplus}$ and $\tilde{m}^{\ominus}$, respectively) are suppressed on locations where no contrast measurements are available. Suppression is achieved by a confidence measure $\kappa \in[0,1]$, and resulting surface representations are free of 'bowing' artifacts. BEATS filling-in does not reveal 'bowing' artifacts, since (i) it employs nonlinear max-diffusion, which permits pulselike injection of filling-in contrasts to achieve uniformly filled-in surface representations (independent of their size), and (ii) BEATS filling-in dose not incorporate a passive decay of activity in the cells of the diffusion layers.

Diffusion layers for confidence-based filling-in are defined as:

$\frac{\mathrm{d} c_{i j}^{\circ}(t)}{\mathrm{d} t}=\left(\tilde{m}_{i j}^{\oplus}-g_{\text {leak }} c_{i j}^{\circ}\right) \kappa_{i j}+\mathcal{K}_{\varepsilon, 0}^{\circ} c_{i j}^{\circ}$
$\frac{\mathrm{d} c_{i j}^{\bullet}(t)}{\mathrm{d} t}=\left(\tilde{m}_{i j}^{\ominus}-g_{\text {leak }} c_{i j}^{\bullet}\right) \kappa_{i j}+\mathcal{K}_{\varepsilon, 0}^{\bullet} c_{i j}^{\bullet}$

$\kappa$ is a monotonic and bounded function of boundary activity, e.g. $\kappa_{i j}=1-\exp \left(\gamma_{w} w_{i j}\right)$. Notice that standard filling-in is obtained for $\kappa_{i j}=1 \forall i, j$. Confidence-based filling-in uses a single set of boundaries $w$, which is computed here by spatially blurring contours and cofftours (Eq. (9)):

$w=\left(w^{\circ}+w^{\bullet}\right) \otimes \mathscr{L}_{\mathrm{w}}$

Both, standard filling-in and confidence-based filling-in use heat diffusion (diffusion operators $\mathcal{K}_{\varepsilon, 0}^{\circ}$ and $\mathcal{K}_{\varepsilon, 0}^{\bullet}$ ), that is $\lambda=0$ in Eq. (3). Permeabilities $P^{\circ} \equiv P^{\circ}\left(\Gamma^{\circ}\right)$ and $P^{\bullet} \equiv P^{\bullet}\left(\Gamma^{\bullet}\right)$ are instantiated by defining (see Eq. (4))

$\Gamma_{i j p q}^{\circ} \equiv \Gamma_{i j p q}^{\bullet}=w_{i j}+w_{p q}$ 
Table 4

Parameter values for confidence-based filling-in

\begin{tabular}{|c|c|c|c|}
\hline Parameter & Value & Equation & Description \\
\hline$\varepsilon$ & 750 & 3 & $\begin{array}{l}\text { contour/cofftour diffusion } \\
\text { slow down gain }\end{array}$ \\
\hline$\lambda$ & 0 & 3 & Laplacian diffusion \\
\hline$\Theta_{w}$ & 0.025 & 9 & contour/cofftour threshold \\
\hline$\beta_{w}$ & 25 & 9 & $\begin{array}{l}\text { contour/cofftour saturation } \\
\text { constant }\end{array}$ \\
\hline$\sigma_{w}$ & 0.25 & 16 & Blurring constant \\
\hline$g_{\text {leak }}$ & 0.001 & 15 & Leakage conductance \\
\hline
\end{tabular}

Specific parameter values are not crucial for our conclusions, as long as they stay within the same order of magnitude.

At steady-state, Eq. (15) can be expressed in matrix notation (with matrix coefficients denoting lateral interactions), and hence filled-in brightness and darkness, respectively, can be computed by matrix inversion (further details are given in Neumann et al., 2001). The output $S^{\circ} \equiv\left[c^{\circ}\right]^{+}$and $S^{\bullet} \equiv\left[c^{\bullet}\right]^{+}$is obtained by half-wave rectified activities of layer cells, and perceptual activities are obtained through Eq. (14). Parameter values for confidence-based filling-in are given in Table 4.

\section{Limitations of filling-in}

Most filling-in models (and also other models for brightness perception) reveal some artifacts which are illustrated in Fig. 7, and which are detailed below.

\subsection{Activity trapping}

A first type of activity trapping occurs if filling-in contrasts spatially overlap with boundary activity: since boundaries act to locally decrease activity exchange between adjacent cells, filled-in activities remain large at boundaries, and get continuously smaller when moving away from them. Activity trapping gets particularly pronounced with high contrast boundaries (Fig. 8), and occurs both with standard-filling, and confidence-based filling-in. The reason is that identical boundary sets are used for the brightness and the darkness diffusion layer (Fig. 9), which must be blurred in order to achieve symmetric alignment 'between' the interior and the exterior of surfaces (c.f. Eq. (16)).

With BEATS filling-in, filling-in contrasts do not overlap with boundaries (Fig. 5), since different boundaries are used for the brightness and the darkness diffusion layer (cofftours and contours, respectively). As a consequence, the latter type trapping cannot occur.

Nevertheless, a second source of trapping can be the presence of boundary webs (Grossberg \& Mingolla, 1987; Pessoa et al., 1995) inside surfaces, as generated, for example, by smooth luminance gradients. If boundary activity associated with such webs is sufficiently high, diffusion will get 'frozen'. The latter type of trapping also affects BEATS filling-in.

\subsection{Fogging}

Fogging refers to a loss of perceived contrast as a consequence of activity exchange between adjacent surface representations. This situation typically occurs for two reasons. First, activity may be exchanged across boundaries with small amplitudes. Second, activity may be exchanged through boundary gaps (or holes), where activity leaks through to adjacent surface representations. To fix such gaps, small-scale grouping mechanisms were proposed, in order to render filling-in areas 'watertight' (e.g. Gove et al., 1995; Grossberg et al., 1995; Mingolla et al., 1999). Alternatively, boundaries can be blurred in a way that holes are 'smeared up'. Doing so, however, can lead to activity trapping (due to a now increased overlap with filling-in contrasts), and also to the blurring problem.

BEATS filling-in tackles the problem of leaky boundaries by the interaction zone around contours and cofftours, where brightness activity and darkness activity can undergo mutual inhibition (Eqs. (10) and (11)). Activity diffusion across boundaries is avoided by making brightness (darkness) activity negative-valued at boundary locations, since nonlinear diffusion can only propagate positive values.

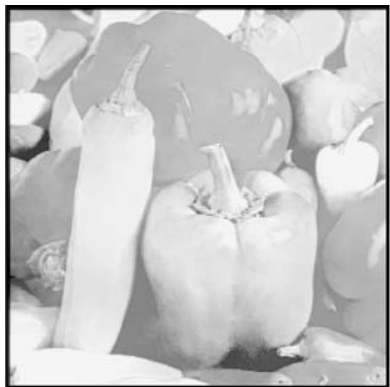

fogging

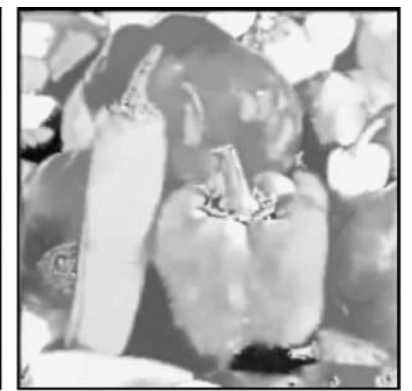

blurring

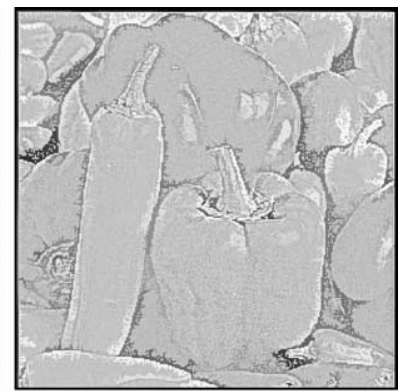

trapping

Fig. 7. Filling-in artifacts. 'Fogging', 'blurring', and an extreme example of 'trapping' is illustrated with the image Peppers (original shown in Fig. 11). All three images were produced with BEATS filling-in, by blurring, and changing parameter values, respectively, of contours and cofftours. 


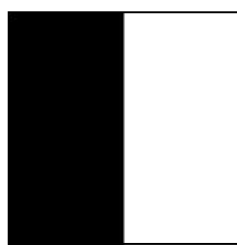

luminance (input) $256 \times 256$ pixel

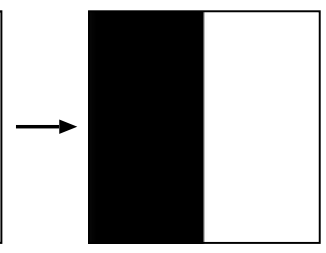

BEATS filling-in (600 time steps)

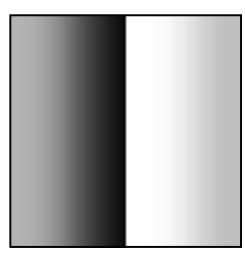

confidence-based filling-in
Fig. 8. Activity trapping. The left image shows a luminance step which served as input image (size $256 \times 256$ pixel). BEATS filling-in does not reveal activity trapping (middle image), whereas confidence-based filling does (right image). The last two images show individually normalized perceptual activities.

\subsection{Blurring}

Methods for boundary detection usually increase the degree of spatial uncertainty of boundaries (e.g. Neumann, 1994). However, when the detected boundaries are too blurry, then edges of the corresponding filled-in surface
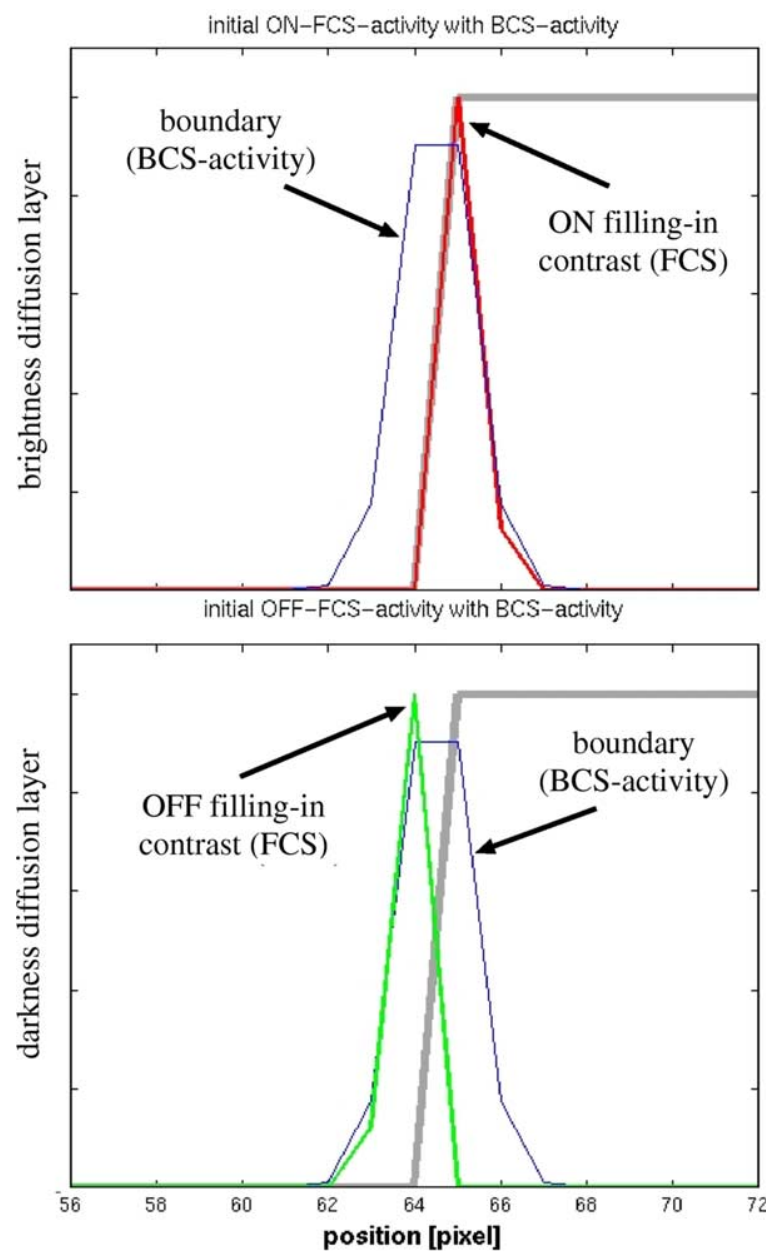

Fig. 9. Initial state of diffusion layers with standard filling-in. Both diffusion layers make use of the same boundary $w$. As a consequence, boundaries overlap with initial filling-in contrasts. Since boundaries act to locally decrease the diffusion rate, initial filling-in contrasts are 'trapped' (c.f. Fig. 8). The luminance profile corresponds to the bold gray curves. representation will appear also more blurred than they originally were. The blurring problem seems to be of particular importance with multi-scale approaches, and one can observe it in the output of various filling-in architectures (e.g. Gove et al., 1995; Grossberg et al., 1995; Sepp \& Neumann, 1999; Mingolla et al., 1999). The blurring problem can also occur as an intermediate situation between trapping and fogging, if a partial activity exchange across boundaries takes place.

In principle there exist two ways for counteracting this problem, namely re-sharpening of blurred boundaries (e.g. by center-surround mechanisms), or ensuring spatial accuracy during the boundary detection process. BEATS filling-in in its present (single-scale) form makes use of two sets of high-resolution boundary maps to overcome this problem. Nevertheless, in this way it is less robust against noise compared with multi-scale approaches for boundary detection.

\subsection{Recovering absolute levels of perceived luminance}

Only few approaches to brightness perception incorporate neurophysiologically satisfying solutions to the problem of how to go from contrast measurements to the absolute levels of perceived luminance of a surface (e.g. Arrington, 1996). A standard technique is to band-pass filter the visual input over multiple scales, and subsequently adding luminance information to recover absolute luminance levels (e.g. Hong \& Grossberg, 2004; Neumann, 1996; Pessoa et al., 1995). We instead propose a multiplexed retinal code, which is generated from contrast responses by modulating ON-cell (OFF-cell) responses with local brightness (darkness) (Fig. 10). Multiplexed retinal responses represent

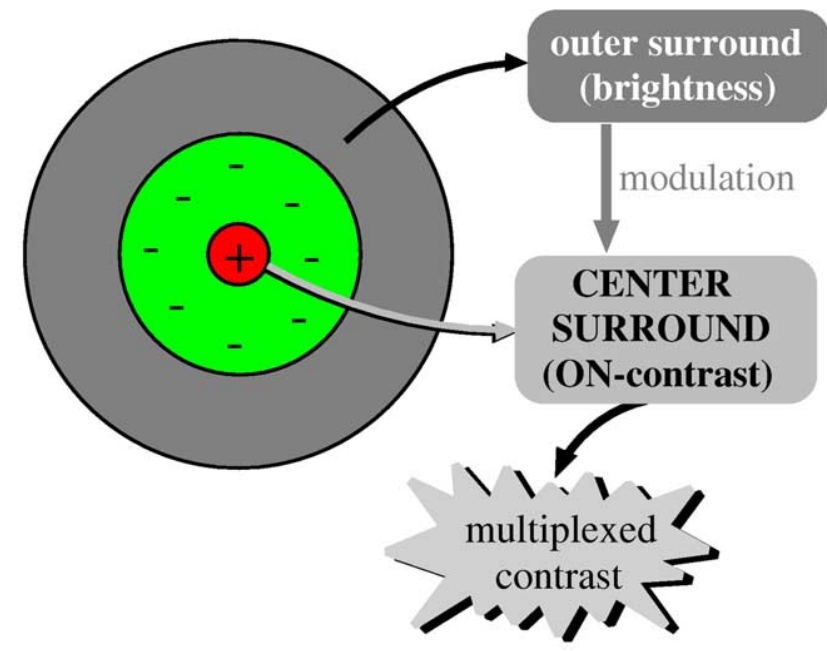

Fig. 10. Creating a multiplexed retinal code. An excitatory center and an inhibitory surround generate an ON-response. An outer surround subsequently modulates ON-contrast amplitudes according to local intensity. In this way a multiplexed ON-response is generated, which encodes local brightness. In an analogous fashion, OFF-type responses are modulated by local darkness. 
filling-in contrasts, and below we show that single-scale information is sufficient to recover perceived luminance maps with natural images.

\section{Simulation results}

The performance of BEATS filling-in ('beats-FI') and confidence-based filling-in ('confidence-FI') were evaluated by simulation experiments. The luminance maps which served as input images are shown in Fig. 11. Filled-in results (i.e. perceived luminance) are shown in Figs. 12 and 13 for beats-FI and confidence-FI, respectively.

The edges of the surface representations generated with beats-FI are sharper than they actually were in the input images. This is a consequence of the nonlinear diffusion operator, which effectuates deblurring (or deconvolution) of edges, and suppression of luminance gradients, respectively (see the out-of-focus bar in the background of the Lena image). However, different to backward diffusion

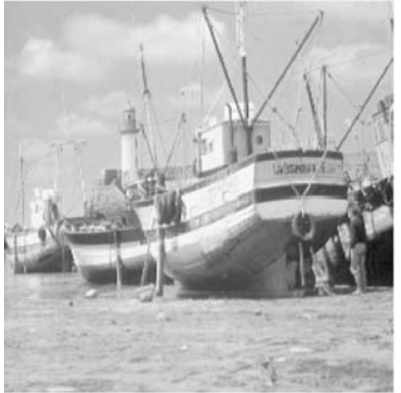

Boats

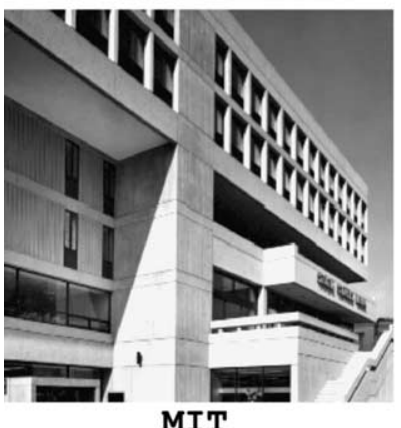

MIT

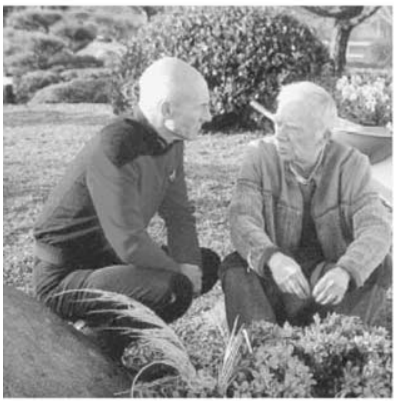

Picard
Lena

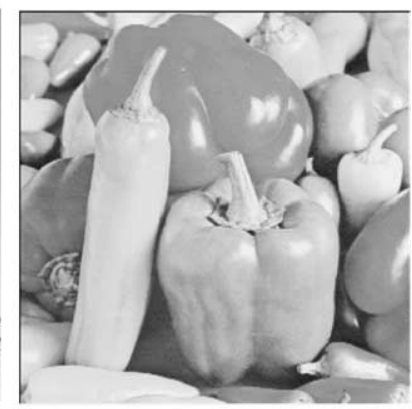

Peppers

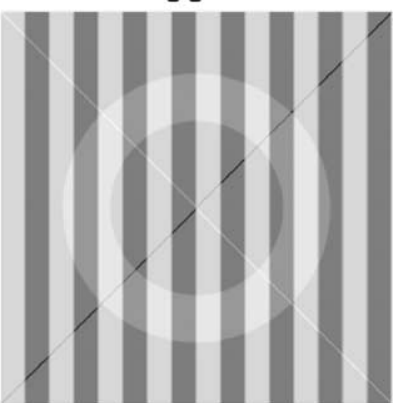

du Buf

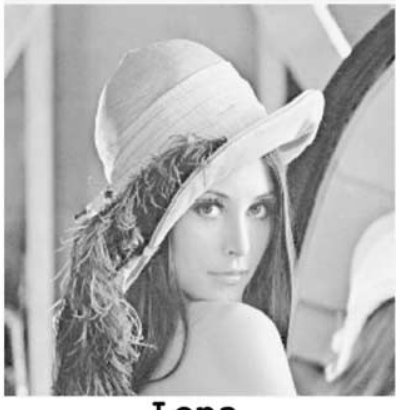

Fig. 11. Input images. Images show luminance. All images were of size $256 \times 256$ pixels with 256 gray levels. For the simulations, the images were normalized such that intensity values lay between zero and one.
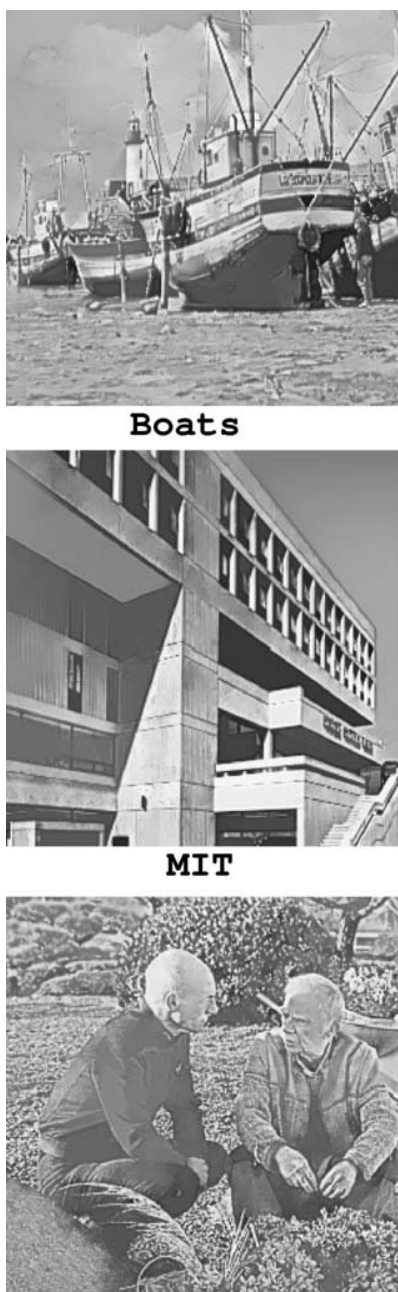

Picard

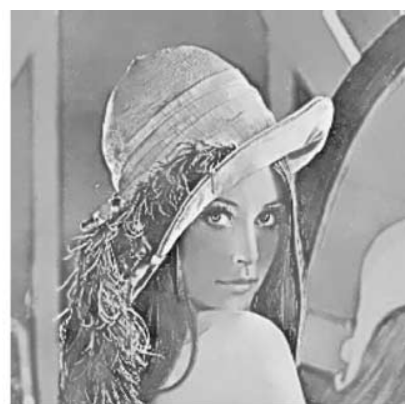

Lena

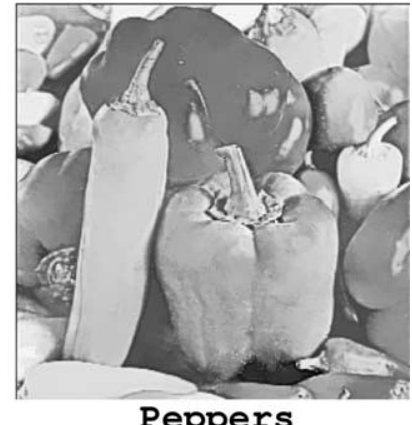

Peppers

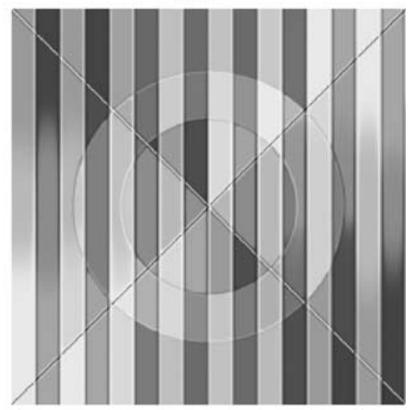

du Buf
Fig. 12. Results for BEATS filling-in. Images show the perceptual activities (perceived luminance). For displaying, all images were individually normalized. Input images are shown in Fig. 11.

approaches (as, e.g. Perona \& Malik, 1990), deconvolution by nonlinear max-diffusion does not involve numerical instabilities. Another observation with beats-FI is that there is a loss of contrast in perceived luminance. This is because retinal filling-in contrasts tend to be higher at feature junctions (e.g. corners), and all cells representing a surface will adopt these activity values due to nonlinear diffusion. Conversely, higher activities at feature junctions do not represent a problem with a linear diffusion mechanism, since they are simply averaged out.

With confidence-FI we can observe a certain degree of blurring in the sense of a partial exchange of activities across boundaries, what leads to 'washing out' of fine structured details (as it is most obvious with the image Boats and the image Picard, where persons' heads appear to be self-luminous). The reason lies in corresponding small boundary activities.

To prevent activity diffusion across boundaries, one could in principle increase the gain of boundary signals. 


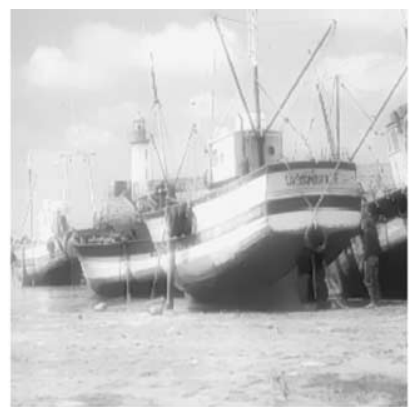

Boats

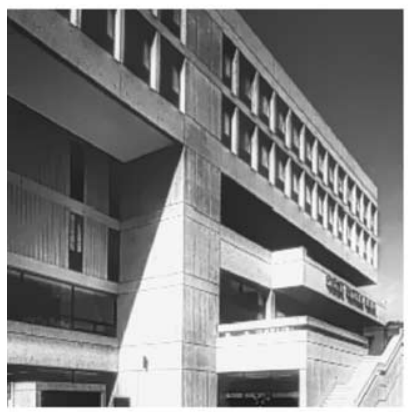

MIT

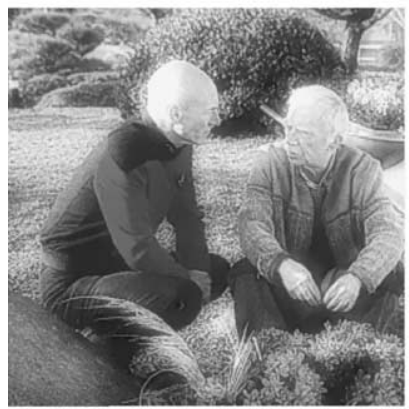

Picard

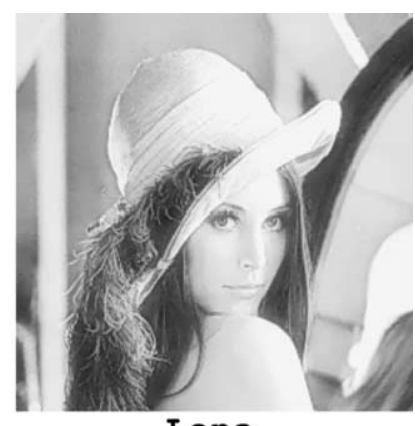

Lena

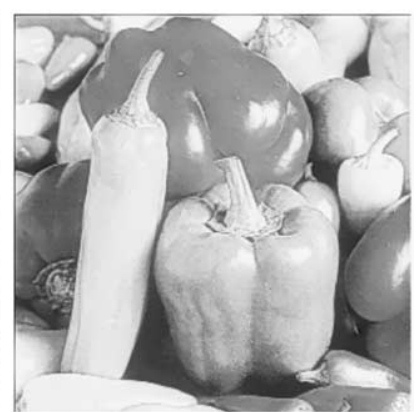

Peppers

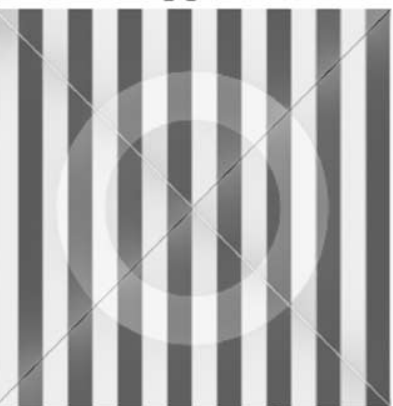

du Buf
Fig. 13. Results for confidence-based filling-in. Images show the perceptual activities (perceived luminance). For displaying, all images were individually normalized. Input images are shown in Fig. 11.

In this way fine structure is preserved, but the trapping problem due to contrast overlap gets more pronounced, what causes a decrement in perceptual contrasts (as exemplified with Fig. 7). As an alternative, one could use contours and cofftour with confidence-based filling-in. Although blurring virtually disappears in the latter case, one must increase boundary activity sufficiently to prevent fogging. Doing so, however, increases activity trapping due to boundary webs.

The principal advantage of confidence-FI over beats-FI are the perceived contrasts: confidence-FI has the tendency to even exaggerate contrasts in filled-in surface representations.

\section{Discussion and conclusions}

With the present article we presented an advance to filling-in theory. We proposed a novel mechanism for encoding luminance information in contrast responses of retinal ganglion cells: retinal ON-responses are modulated according to local brightness levels, and OFF-responses according to local darkness levels ('multiplexed retinal code'). An outer surround is used to locally measure these intensity levels. The outer surround is located beyond the classical center/surround receptive field of retinal ganglion cells. The multiplex code represents a neurophysiologically plausible mechanism for addressing the problem of how the visual system recovers absolute luminance levels from contrasts. The solution is plausible since it represents a consistent interpretation of the findings from Li et al. (1991, 1992). The latter authors conjectured that the outer surround plays a role in transmitting luminance information. Furthermore, recent evidence from measuring EEG-activity in humans strongly favors a fast and low-level mechanism for encoding luminance information over mid-level or higher-level processing (McCourt \& Foxe, 2004). By means of filling-in, perceived luminance can directly be recovered from the multiplex code. Consequently, we hypothesize that two retinal pathways are involved in luminance perception: one for detecting boundaries, and another for recovering the perceived luminance of surfaces. This distinction between 'retinal boundary contrasts' and 'filling-in contrasts' is not included in other filling-in models proposed up to the present. We demonstrated by simulations with real-world images that single-scale information is sufficient for recovering absolute levels of perceived luminance. In other words, it seems that images can be recovered from their boundaries (or contrasts). This should be compared with the widespread notion that a multi-scale decomposition of the visual input is necessary in order to create a faithful representation of the visual input. One advantage of multiscale approaches over the single-scale scheme proposed in this paper is that the former are more robust against noise. Furthermore, within the context of filling-in, multi-scale multiplex contrasts could presumably accelerate the fillingin process (c.f. Sepp \& Neumann, 1999). A further hypothesis is that odd symmetric features on the finest scale eventually lead to surface representations, whereas grosser scales trigger representations of smooth luminance gradients (or shading, Keil, Cristóbal, \& Neumann, 2005).

As a second contribution of our paper we identified some shortcomings with present filling-in models: the blurring problem, the fogging problem, and the trapping problem (Section 6 and Fig. 7). As an solution to those problems we proposed BEATS filling-in, which is based on nonlinear diffusion mechanisms.

Specifically, BEATS filling-in employs different boundaries in each diffusion layer ('contours' and 'cofftours'), and avoids in this way the overlap of boundaries and contrasts for filling-in (activity trapping, see Section 6.1). Since these boundaries correspond to high spatial frequencies, boundary allocation is accurate, and the blurring problem is reduced (see Section 6.3). The fogging problem (Section 6.2) is addressed by using a nonlinear diffusion 
mechanism for filling-in, which cannot propagate negative activity values.

We subsequently compared the results of BEATS fillingin with confidence-based filling-in: BEATS filling-in maintains the fine structured details of surface representations, but reveals a decrease in contrast of perceived activity. Confidence-based filling-in, on the other hand, attenuates high spatial frequencies, but enhances contrasts of perceived luminance. Both models are minimally complex in the sense that they cannot be reduced further to obtain the results we presented. Moreover, we omitted an explicit stage for boundary detection for simplicity reasons. Nevertheless, by incorporating such a stage, one could expect that perceived luminance is more faithful to the input, and an increase in the explanatory power of our models (we did not make any attempt to simulate psychophysical data).

The ultimate goal of understanding human luminance perception is a model which both predicts psychophysical data, while having a robust performance when processing real-world images. Such a model can even be expected to discount noise which was present in the input, and/or enhance the subjective appearance of the input. At the present, only few such models are available (e.g. Hong \& Grossberg, 2004). Our contribution takes a step towards this direction. Since filling-in models were successful in explaining a broad spectrum of psychophysical data (e.g. Arrington, 1994; Arrington, 1996; Gove et al., 1995; Grossberg \& Todorović, 1988; Grossberg, Hwang, \& Mingolla, 2002; Neumann, Pessoa, \& Mingolla, 1998; Pessoa et al., 1995; Pessoa, 1996; Pessoa \& Ross, 2000; Rudd \& Arrington, 2001), we do believe that it is worthwhile to improve those models in a way to make them suitable for image processing tasks.

\section{Acknowledgements}

The authors wish to thank the reviewers, whose comments helped to improve our manuscript. This work has been partially supported by the following grants: Spanish-German Academic Research Collaboration Program (DAAD, Acciones Integradas Hispano-Alemanes 2002/03, Proyecto No. HA 2001-0087); AMOVIP INCODC 961646, TIC2001-3697-C03-02; and, the IM3 medical imaging thematic network from the Instituto de Salud Carlos III.

\section{References}

Arrington, K. (1994). The temporal dynamics of brightness filling-in. Vision Research, 34(24), 3371-3387.

Arrington, K. (1996). Directional filling-in. Neural Computation, 8, 300-318
Berson, D., Dunn, F., \& Takao, M. (2002). Phototransduction by retinal ganglion cells that set the circadian clock. Science, 295, 1070-1073.

Chichilnisky, E., \& Kalmar, R. (2002). Functional asymmetries in ON and OFF ganglion cells of primate retina. The Journal of Neuroscience, 22(7), 2737-2747.

Cleland, B., Levick, W., \& Sanderson, K. (1973). Properties of sustained and transient ganglion cells in the cat retina. Journal of Physiology (London), 228, 649-680.

Cohen, M., \& Grossberg, S. (1984). Neural dynamics of brightness perception: Features, boundaries, diffusion and resonance. Perception and Psychophysics, 36, 428-456.

Davey, M., Maddess, T., \& Srinivasan, M. (1998a). The spatiotemporal properties of the Craik-O'Brien-Cornsweet effect are consistent with 'filling-in'. Vision Research, 38, 2037-2046.

Davey, M., Srinivasan, M., \& Maddess, T. (1998b). The Craik-O'BrienCornsweet in honeybees. Naturwissenschaften, 85, 73-75.

du Buf, J., \& Fischer, S. (1995). Modeling brightness perception and syntactical image coding. Optical Engineering, 34(7), 1900-1911.

Edwards, D., Heitler, W., Leise, E., \& Friscke, R. (1991). Postsynaptic modulation of rectifying electrical synaptic inputs to the lg escape command neuron in crayfish. The Journal of Neuroscience, 11, 2117-2129.

Edwards, D., Yeh, S.-R., \& Krasne, F. (1998). Neuronal coincidence detection by voltage-sensitive electrical synapses. Proceedings of the National Academy of Sciences USA, 9(12), 7145-7150.

Gerrits, H., de Haan, B., \& Vendrik, A. (1966). Experiments with retinal stabilized images. Relations between the observations and neural data. Vision Research, 6, 427-440.

Gerrits, H., \& Vendrik, A. (1970). Simultaneous contrast, filling-in process and information processing in man's visual system. Experimental Brain Research, 11, 411-430.

Gilchrist, A., Kossyfidis, C., Bonato, F., Agostini, T., Cataliotti, J., Li, X., et al. (1999). An anchoring theory of lightness perception. Psychological Review, 106(4), 795-834.

Gove, A., Grossberg, S., \& Mingolla, E. (1995). Brightness perception, illusory contours, and corticogeniculate feedback. Visual Neuroscience, $12,1027-1052$.

Grossberg, S. (1997). Cortical dynamics of three-dimensional figureground perception of two-dimensional pictures. Physiological Review, 104, 618-658.

Grossberg, S., Francis, G., \& Mingolla, E. (1994). Cortical dynamics of feature binding and reset: Control of visual persistence. Vision Research, 34, 1089-1104.

Grossberg, S., \& Howe, P. (2003). A laminar cortical model of stereopsis and three-dimensional surface perception. Vision Research, 43, 801-829.

Grossberg, S., Hwang, S., \& Mingolla, E. (2002). Thalmocortical dynamics of the McCollough effect: Boundary-surface alignment through perceptual learning. Vision Research, 42, 1259-1286.

Grossberg, S., \& Mingolla, E. (1985). Neural dynamics of form perception: boundary completion, illusory figures, and neon color spreading. Psychological Review, 92(2), 173-211.

Grossberg, S., \& Mingolla, E. (1987). Neural dynamics of surface perception: boundary webs, illuminants, and shape-from-shading. Computer Vision, Graphics, and Image Processing, 37, 116-165.

Grossberg, S., Mingolla, E., \& Williamson, J. (1995). Synthetic aperture radar processing by a multiple scale neural system for boundary and surface representation. Neural Networks, 8(7-8), 1005-1028.

Grossberg, S., \& Pessoa, L. (1998). Texture segregation, surface representation, and figure-ground separation. Vision Research, 38, 2657-2684.

Grossberg, S., \& Todorović, D. (1988). Neural dynamics of 1-d and 2-d brightness perception: A unified model of classical and recent phenomena. Perception and Psychophysics, 43, 241-277.

Hong, S., \& Grossberg, S. (2004). A neuromorphic model for achromatic and chromatic surface representation of natural images. Neural Networks, 17(5-6), 787-808. 
Hubel, D., \& Wiesel, T. (1962). Receptive fields, binocular interaction and functional architecture in the cat's visual cortex. Journal of Physiology, London, 160, 106-154.

Hubel, D., \& Wiesel, T. (1968). Receptive fields and functional architecture of monkey striate cortex. Journal of Physiology, London, 195, 214-243.

Kaplan, E., Lee, B., \& Shapley, R. (1990). New views of primate retinal function. Progress in Retinal Research, 9, 273-336.

Kaplan, E., Purpura, K., \& Shapley, R. (1987). Contrast affects the transmission of visual information through the mammalian lateral geniculate nucleus. Journal of Physiology (London), 391, 267-288.

Kaplan, E., \& Shapley, R. (1982). X and y cells in the lateral geniculate nucleus of macaque monkeys. Journal of Physiology, 330, 125-143.

Keil, M. (2003). Neural architectures for unifying brightness perception and image processing. $\mathrm{PhD}$ thesis, Universität Ulm, Faculty for Computer Science, Ulm, Germany.

Keil, M., Cristóbal, G., Neumann, H. (2005). Gradient representation and perception in the early visual system-a novel account to Mach band formation. Vision Research, (in revision).

Keil, M., Neumann, H. (2001). Modeling the dynamics of filling-in: reset circuits, aftereffects and the medial axis transform. In Proceedings of the Fifth International Conference on Cognitive and Neural Systems, Boston. Center for Adaptive Systems and Department of Cognitive and Neural Systems Boston University.

Kelly, F., \& Grossberg, S. (2000). Neural dynamics of 3-D surface perception: figure-ground separation and lightness perception. Perception and Psychophysics, 62, 1596-1619.

Kinoshita, M., \& Komatsu, H. (2001). Neural representations of the luminance and brightness of a uniform surface in the macaque primary visual cortex. Journal of Neurophysiology, 86, 2559-2570.

Knau, H., \& Spillman, L. (1997). Brightness fading during Ganzfeld adaptation. Journal of the Optical Society of America A, 14(6), 1213-1222.

Komatsu, H., Murakami, I., \& Kinoshita, M. (1996). Surface representations in the visual system. Cognitive Brain Research, 5, 97-104.

Kuffler, S. (1953). Discharge patterns and functional organization of mammalian retina. Journal of Neurophysiology, 16, 37-68.

Li, C.-Y., Pei, X., Zhou, Y.-X., \& von Mitzlaff, H.-C. (1991). Role of the extensive area outside the $\mathrm{X}$-cell receptive field in brightness information transmission. Vision Research, 31(9), 1529-1540.

Li, C.-Y., Zhou, Y.-X., Pei, X., Qiu, F.-T., Tang, C.-Q., \& Xu, X.-Z. (1992). Extensive disinhibitory region beyond the classical receptive field of cat retinal ganglion cells. Vision Research, 32(2), 219-228.

MacEvoy, S., Kim, W., \& Paradiso, M. (1998). Integration of surface information in the primary visual cortex. Nature Neuroscience, 1(7), 616-620.

Mariani, A. (1982). Biplexiform cells: ganglion cells of the primate retina that contact photreceptors. Science, 216, 1134-1136.

Marr, D. (1982). Vision. New York: W.H. Freeman and Company.

Marr, D., \& Hildreth, E. (1980). Theory of edge detection. Proceedings of the Royal Society of London B, 207, 187-217.

McCourt, M. and Foxe, J. (2004). Brightening prospects for 'early' cortical coding of perceived luminance. Journal of Vision, (to appear).

Mingolla, E., Ross, W., \& Grossberg, S. (1999). A neural network for enhancing boundaries and surfaces in synthetic aperture radar images. Neural Networks, 12, 499-511.

Neumann, H. (1994). An outline of a neural architecture for unified visual contrast and brightness perception. Technical Report CAS/CNS-94003, Boston University Center for Adaptive Systems and Department of Cognitive and Neural Systems.

Neumann, H. (1996). Mechanisms of neural architectures for visual contrast and brightness perception. Neural Networks, 9(6), 921-936.
Neumann, H., Pessoa, L., \& Hansen, T. (2001). Visual filling-in for computing perceptual surface properties. Biological Cybernetics, 85, 355-369.

Neumann, H., Pessoa, L., \& Mingolla, E. (1998). A neural architecture of brightness perception: nonlinear contrast detection and geometrydriven diffusion. Image and Vision Computing, 16, 423-446.

Paradiso, M., \& Hahn, S. (1996). Filling-in percepts produced by luminance modulation. Vision Research, 36, 2657-2663.

Paradiso, M., \& Nakayama, K. (1991). Brightness perception and filling-in. Vision Research, 31(7/8), 1221-1236.

Passaglia, C., Enroth-Cugell, C., \& Troy, J. (2001). Effects of remote stimulation on the mean firing rate of cat retinal ganglion cells. Journal of Neuroscience, 21, 5794-5803.

Perona, P., \& Malik, J. (1990). Scale-space and edge detection using anisotropic diffusion. IEEE Transactions on Pattern Analysis and Machine Intelligence, 12(7), 629-639.

Pessoa, L. (1996). Mach-band attenuation by adjacent stimuli: experiment and filling-in simulations. Perception, 25(4), 425-442.

Pessoa, L., Mingolla, E., \& Neumann, H. (1995). A contrast- and luminance-driven multiscale network model of brightness perception. Vision Research, 35(15), 2201-2223.

Pessoa, L., \& Neumann, H. (1998). Why does the brain fill-in? Trends in Cognitive Sciences, 2, 422-424.

Pessoa, L., \& Ross, W. (2000). Lightness from contrast: A selective integration model. Perception and Psychophysics, 62(6), 1160-1181.

Pessoa, L., Thompson, E., \& Noë, A. (1998). Finding out about filling-in: A guide to perceptual completion for visual science and the philosophy of perception. Behavioral and Brain Sciences, 21, 723-802.

Ross, W., Grossberg, S., \& Mingolla, E. (2000). Visual cortical mechanisms of perceptual grouping: interacting layers, networks, columns and maps. Neural Networks, 13(6), 571-588.

Rossi, A., \& Paradiso, M. (1999). Neural correlates of perceived brightness in the retina, lateral geniculate nucleus, and striate cortex. The Journal of Neuroscience, 193(14), 6145-6156.

Rossi, A., Rittenhouse, C., \& Paradiso, M. (1996). The representation of brightness in primary visual cortex. Science, 273, 1391-1398.

Rudd, M., \& Arrington, K. (2001). Darkness filling-in: a neural model of darkness induction. Vision Research, 41(27), 3649-3662.

Sasaki, Y., \& Watanabe, T. (2004). The primary visual cortex fills in color. Proceedings of the National Academy of Sciences USA, 52(101), 18251-18256.

Sepp, W., Neumann, H. (1999). A multi-resolution filling-in model for brightness perception. In ICANN99 Conference Publication. Vol. 470, pp 461-466. Ninth International Conference on Artificial Neural Networks.

Troy, J., \& Robson, J. (1992). Steady discharges of X and Y retinal ganglion cells of cat under photopic illuminance. Visual Neuroscience, 9, 535-553.

Walls, G. (1954). The filling-in process. American Journal of Optometry and Archives of American Academy of Optometry, 31, 329-341.

Watanbade, T., \& Sato, T. (1989). Effects of luminance contrast on color spreading and illusory contour in the neon color spreading effect. Perception and Psychophysics, 45, 427-430.

Werblin, F., \& Dowling, J. (1969). Organization of the retina in the mudpuppy. Necturus maculosus. II. Intracellular recording. Journal of Neurophysiology, 32(9), 339-355.

Zaghloul, K., Boahen, K., \& Demb, J. (2003). Different circuits for ON and OFF retinal ganglion cells cause different contrast sensitivities. The Journal of Neurosciene, 23(7), 2645-2654.

Zrenner, E., Nelson, R., \& Mariani, A. (1983). Intracellular recordings from a biplexiform ganglion cell in macaque retina, stained with horseradish peroxidase. Brain Research, 262, 181-185. 